\title{
A case of mucosa-associated lymphoid tissue (MALT) lymphoma arising in the accessory parotid gland
} \author{
Kazuo Sakurai ${ }^{3}$ \\ ${ }^{1}$ Department of Surgical Pathology, Fujita Health University, School of Medicine, Aichi, Japan \\ ${ }^{2}$ Second Department of Pathology, Fujita Health University, School of Medicine, Aichi, Japan \\ ${ }^{3}$ Department of Otolaryngology, Fujita Health University, School of Medicine, Aichi, Japan
}

Makoto Urano $^{1}$, Yuka Kiriyama ${ }^{1}$, Masato Abe ${ }^{1}$, Makoto Kuroda ${ }^{1}$, Yoshikazu Mizoguchi ${ }^{2}$ and

Abstract: A 62-year-old female visited our hospital complaining of a swelling in her right cheek. Computed tomography (CT) revealed a well-demarcated solid tumor, $2 \times 2 \mathrm{~cm}$ in size, on the outer side of the right masseter muscle, located apart from the parotid gland. We suspected malignant lymphoma using fine-needle aspiration cytology, and an open biopsy was performed. Histologically, proliferation of atypical small cleaved lymphocytes was seen around the enlarged lymph follicles. Residual salivary duct epithelium was detected within the lesion. In immunohistochemical staining, these tumor cells were positive for CD20, CD79a and bcl-2, but not positive for CD10, CD3, and CD45RO. We diagnosed the tumor as mucosa-associated lymphoid tissue (MALT) lymphoma arising in an accessory parotid gland. According to the histological diagnosis, radiotherapy was done and the patient had complete remission. However, forty-three months after treatment, she had a recurrent tumor in the inguinal lymph node. [Oral Med Pathol 2007; 12: 19-22]

Key words: malignant lymphoma, MALT, accessory parotid gland, anatomy, immunohistochemistry

Correspondence: Makoto Urano, Department of Surgical Pathology, Fujita Health University, School of Medicine, 1-98 Dengakugakubo, Kutsukake-cho, Toyoake, Aichi, 470-1192, Japan

Phone: +81-562-93-2319, Fax: +81-562-95-3761, E-mail:uranom@fujita-hu.ac.jp

\section{Introduction}

Mucosa-associated lymphoid tissue (MALT) is recognized as that tissue which exists at the site that mediates mucosa and the outside of the body, including gastrointestinal tract, bronchi and lungs, salivary glands and lacrimal glands, etc.

In pathological conditions, it is believed that a chronic persistent inflammation leads to acquired MALT. Examples of acquired MALT are Heicobactor Pylori associated chronic gastritis in the stomach, Hashimoto's thyroiditis in the thyroid, and Sjögren's syndrome in the salivary glands (1).

The concept of MALT lymphoma was initially suggested by Issacson et al (2) in 1983, and the newest WHO classification has placed it as an extra-nodal marginal zone B-cell lymphoma of mucosa-associated lymphoid tissue. About $14 \%$ of MALT lymphoma is considered to occur in the head and neck region (3). Although the human accessory parotid gland is noticed in approximately $20 \%$ to $56 \%$ of autopsy cases $(4,5,6)$ (Fig1), a tumor, especially a malignant

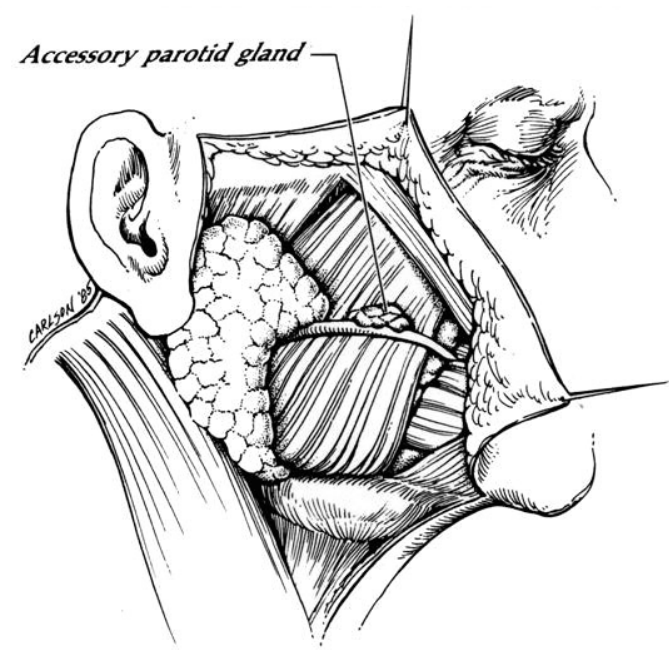

Typical position of accessory parotid gland along course of Stensen's duct. Note accessory gland's complete separation from parotid gland.

Fig. 1. Anatomical state of an accessory parotid gland. (from reference \# 5 Batsakis JG, courtesy of Annals Otology Rhinology Laryngology) 
lymphoma, arising in the parotid gland is extremely rare.

We report a rare case of MALT lymphoma arising in the accessory parotid gland of a 62-year-old female and describe the anatomical features of an accessory parotid gland.

\section{Case report}

A 62-year-old female visited our hospital complaining of a swelling in her right cheek. She had no pain and no facial paralysis. There was no specific past history, and the serological abnormalities of autoantibodies associated with Sjögren's syndrome were not detected.

CT examination revealed a well-demarcated solid tumor, $2 \times 2 \mathrm{~cm}$ in size, on the outer side of the masseter muscle (Fig. 2). Radiologically, the tumor was separated from the parotid gland. We suspected malignant lymphoma using fine needle aspiration cytology and an open biopsy was performed. The tumor was excised piece by piece because it adhered to the Stensen's duct and was difficult to remove.

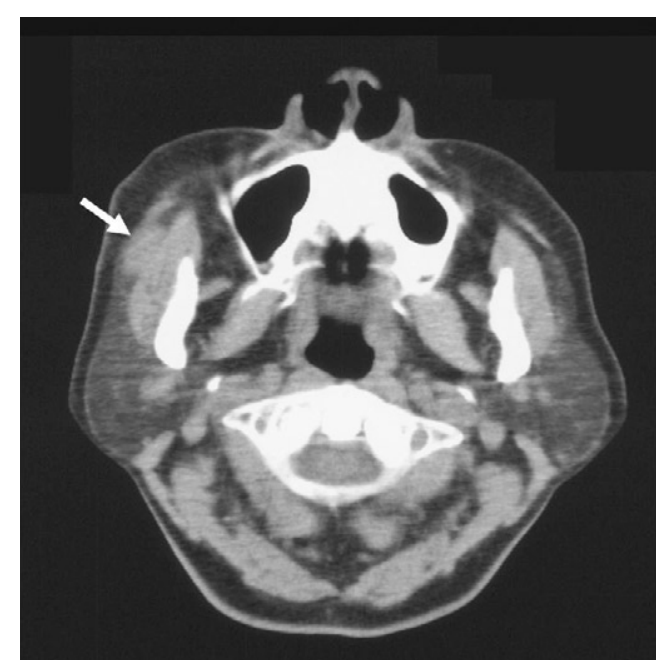

Fig. 2. Computed tomography. A well demarcated solid tumor existed on the outer side of the right masseter muscle (arrow).

After the diagnosis, radiotherapy (24 Gy) was done and the patient had complete remission. However, forty-three months after treatment, she had a recurrent tumor in the inguinal lymph node, and she was treated with radiotherapy (50 Gy) and Rituximab. The patient has been free of disease for twelve months.

\section{Pathological findings}

In the fragmented small pieces of biopsied specimen, various sized lymph follicles lacking in tingible body macrophages existed (Fig. 3a). Among the enlarged follicles, the tumor showed a marginal zone proliferation pattern (Fig. 3b). Atypical small cleaved lymphocytes, which were regarded as centrocyte-like cells, invaded the duct epithelium and formed lymphoepithelial lesions (LELs) to some degree (Fig. 3c). In some of the follicles, tumor cells replaced the existing follicularcenters and made a "follicularcolonization" pattern. Plasma cell differentiation of the tumor cells was not conspicuous. Around the periphery of the lesion, tumor cells infiltrated into surrounding adipose tissue.

Immunohistochemical staining using the labeled streptavidin-biotin (LSAB) method was performed. The primary antibodies used are listed in Table 1. In immunohistochemical staining, these tumor cells were positive for CD20 (Fig. 4a), CD79a and bcl-2, predominantly positive for $\lambda$ light chain (Fig. 4b) rather than $x$ light chain and
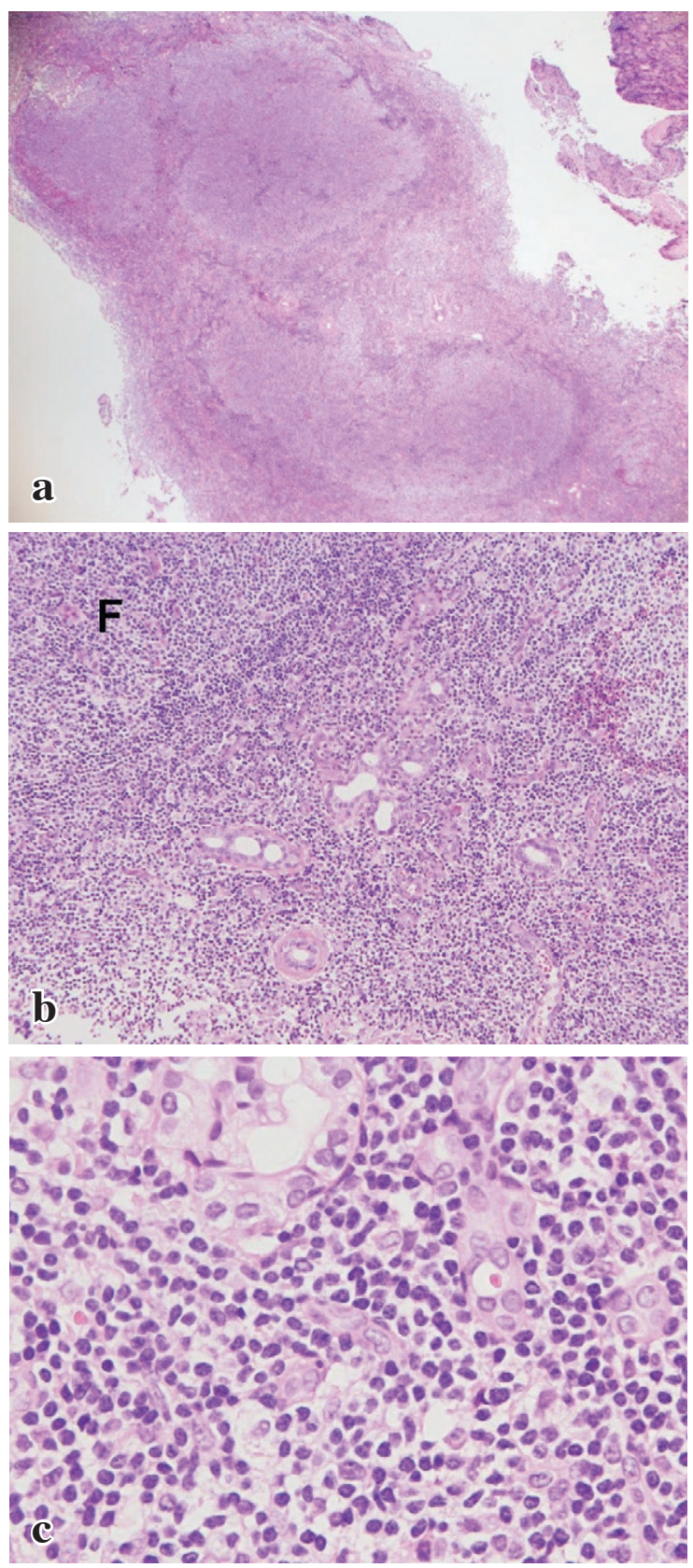

Fig. 3. Histopathology of MALT lymphoma arising in accessory parotid gland. Hematoxylin and eosin (HE) stain. a) Various-sized lymph follicles lacking in tingible body macrophages existed ( $\times 20)$. b) Among the enlarged follicles, the tumor showed marginal zone proliferation pattern (F: follicular center) $(\times 100)$. c) The tumor cells invaded into the duct epithelium and formed lymphoepithelial lesions $(\times 400)$ 
Table 1. Antibodies used in study

\begin{tabular}{llll}
\hline Antibody (clone) & Source & Dilution & Clonality \\
\hline Cytokeratin (CAM5.2) & Becton Dickinson, USA & $(1: 20)$ & Monoclonal \\
CD20 (L26) & DAKO, Glostrup, Denmark & $(1: 800)$ & Monoclonal \\
CD79a (JCB117) & DAKO, Glostrup, Denmark & $(1: 400)$ & Monoclonal \\
CD10 & Novocastra, Newcastle, UK & $(1: 50)$ & Monoclonal \\
bcl-2 (124) & DAKO, Glostrup, Denmark & $(1: 200)$ & Monoclonal \\
$\lambda$ - light chain & DAKO, Glostrup, Denmark & $(1: 40000)$ & Polyclonal \\
$x$ - light chain & DAKO, Glostrup, Denmark & $(1: 20000)$ & Polyclonal \\
CD45RO (UCHL1) & DAKO, Glostrup, Denmark & $(1: 100)$ & Monoclonal \\
CD3 & DAKO, Glostrup, Denmark & $(1: 200)$ & Polyclonal \\
\hline
\end{tabular}

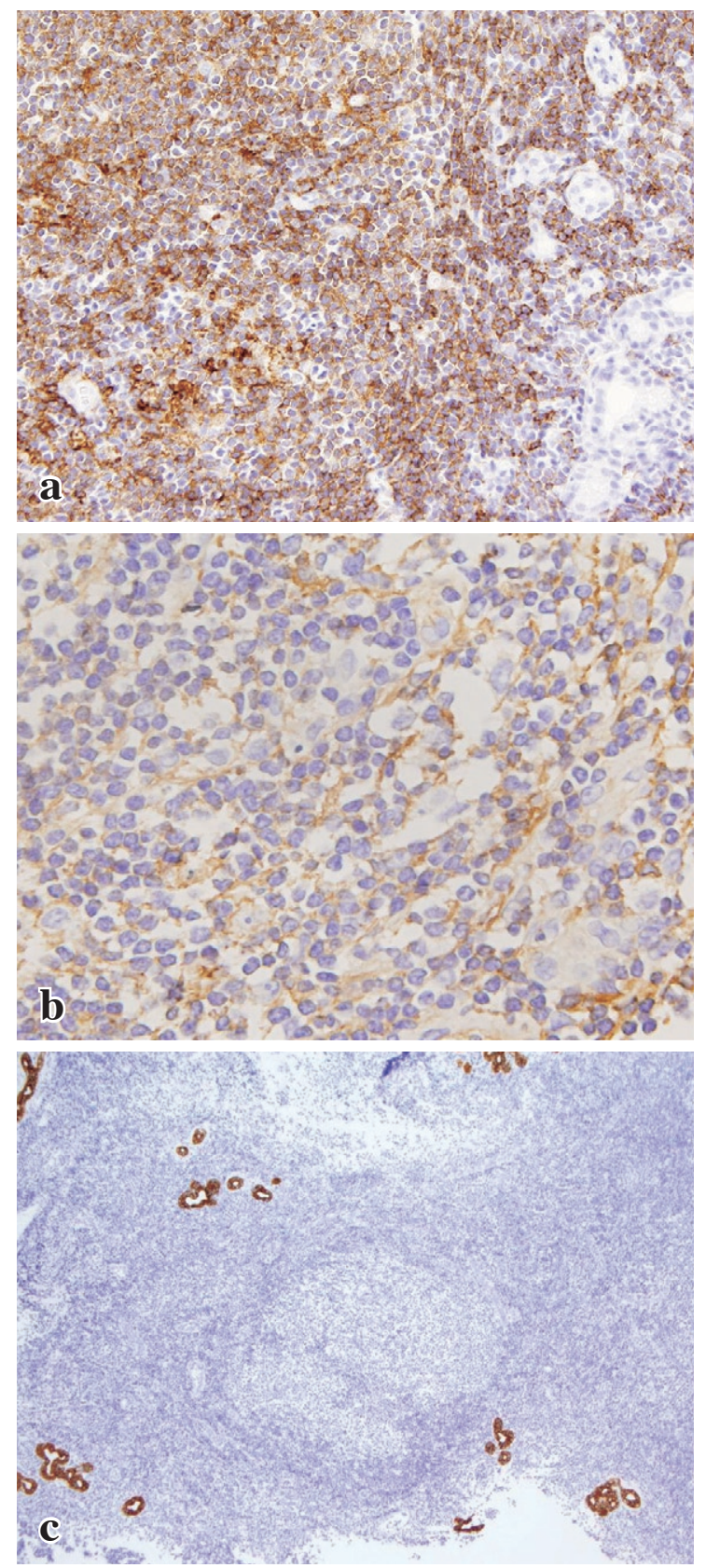

Fig. 4. Immunohistochemistry of MALT lymphoma arising in accessory parotid gland for (a) CD20 (× 200), (b) predominant proportion of $\lambda$ light chain $(\times 400)$, and $(\mathbf{c})$ cytokeratin $(\times 20)$. Immunoperoxidase stain, hematoxylin counterstain. a) The tumor cells were positive for CD20. b) The tumor cells were positive for $\lambda$ light chain. c) The residual duct epithelium was clearly detected within the lesion by cytokeratin staining. negative for CD45RO, CD3 and CD10. The residual duct epithelium was clearly detected within the lesion by cytokeratin staining (Fig. 4c).

The recurrent tumor of the inguinal lymph node showed the same histological findings as nodal marginal zone B cell lymphoma, and an analysis of the tumor tissue detected a $t$ $(14 ; 18)$ gene translocation.

\section{Discussion}

Lymphoid proliferations of the salivary glands include several conditions which are non-neoplastic and neoplastic. The former are benign lymphoepithelial cyst, cystic lymphoid hyperplasia and lymphoepithelial sialadenitis; the latter include MALT lymphoma, follicular lymphoma, diffuse large B cell lymphoma and other types of malignant lymphomas (7).

Primary non-Hodgkin lymphomas arising in the parotid gland are rare, accounting for only about $5 \%$ of all primary extranodal non-Hodgkin lymphomas and $2 \%$ of all salivary gland tumors (8). To our knowledge, this is the first Englishlanguage case report of MALT lymphoma arising in the accessory parotid gland.

In cases of MALT lymphoma of the salivary glands, $10 \%$ to $45 \%$ of these develop extrasalivary lymphoma after long intervals (7).

The current case also had a recurrence of marginal zone lymphoma in the inguinal lymph node after forty-three months of initial therapy.

A tumor arising in an accessory parotid gland is rare. In the literature, several cases have been reported, the majority of them were epithelial tumors which were diagnosed as pleomorphic adenoma $(9,10,11,12)$, myoepithelioma (13, 14), mucoepidermoid carcinoma (15), and squamous cell carcinoma (16).

There are also reports of very rare cases of malignant lymphomas (17). Approximately 50\% all these cases are malignant (18). Fujimura et al (17) reported two cases of non-Hodgkin lymphoma in the accessory parotid gland. One was follicular lymphoma, grade 1 , and the other was diffuse medium lymphoma, B cell type. Both patients had a painless mass in the cheek, and there were no obvious differences between them and our patient in the clinical features. It is difficult to distinguish MALT lymphoma and other types of 
malignant lymphoma without pathological diagnosis.

Frommer (4) described anatomical features of the accessory parotid gland in detail. There are two types of anterior extension of the parotid gland, one is "facial process" which directly attached to the main gland. The other one is "detached glandular mass" or "accessory parotid gland" which completely separated from the main gland. The average distance of separated accessory parotid glands from the anterior edge of the main gland was $6.0 \mathrm{~mm}$. Histologically, there was no significant difference between the main gland and the accessory parotid gland.

From a therapeutical point of view, the tumors arising in the accessory parotid gland present special challenges in terms of surgical procedures.

Because they exist adjacent to or adhere to the Stensen's duct, it is often hard to remove them from the duct without sacrificing it. In the current case, surgeons excised the tumor piece by piece in order not to damage Stensen's duct, and according to histological diagnosis, radiotherapy was done. It is important not to injure the branches of the facial nerve when excising these tumors locally (19).

When seeing cheek region masses, we should pay attention to the possibility of tumor of an accessory parotid gland and recognize the nature of the connection of the tumor to Stensen's duct.

\section{Acknowledgments}

The primary author (M. Urano) is grateful to Mr. Chris Neil for English-language assistance and for his encouragement.

\section{References}

1. Ellis GL, Auclair PL. Tumors of the Salivary Glands. Atlas of Tumor Pathology. Third Series. Fascicle 17. Washington DC: AFIP, 1996; 388-389.

2. Issacson P, Wright DH. Malignant lymphoma of mucosaassociated lymphoid tissue. A distinctive type of B-cell lymphoma. Cancer 1983; 52: 1410-1416.

3. Jaffe ES, Harris NL, Stein H and Vardiman JW.(Ed). World Health Organization Classification of Tumours. Pathology and Genetics of Haematopoitic and Lymphoid Tissues. France, Lyon: IARC Press. 2001; 157-160.

4. Frommer J. The human accessory parotid gland: Its incidence, nature, and significance. Oral Surg 1977; 43: 671-676.
5. Batsakis JG. Accessory Parotid Gland. Ann Otol Rhinol Laryngol 1988; 97: 434-435.

6. Toh H, Kodama J, Fukuda J, Rittman B, Mackenzie I. Incidence and histology of human accessory parotid glands. Anat Rec 1993; 236: 586-590.

7. Harris NL. Lymphoid proliferations of the Salivary Glands. Am J Clin Pathol 1999; 111(Suppl.1): S94-S103.

8. Barnes L, Eveson JW, Reichart P and Sidransky D.(Ed). World Health Organization Classification of Tumours. Pathology and Genetics of Head and neck Tumours. France, Lyon: IARC Press. 2005; 277-280.

9. Kronenberg J, Horowitz A, Creter D. Pleomorphic adenoma arising in accessory salivary tissue with constriction of Stensen's duct. J Laryngol Otol 1988; 102: 382-383.

10. Kakulas EG, Smith AC, Sormann G. Pleomorphic adenoma of the accessory parotid gland: case report. J Oral Maxillofac Surg 1994; 52: 867-870.

11. Horii A, Hinjo Y, Nose M, Ozaki M, Yoshida J. Accessory parotid gland tumor: a case report. Auris Nasus Larynx 1997; 24: 105-110.

12. Osborne RF, Purohit MR, Hamilton JS. Pleomorphic adenoma of the accessory parotid gland. Ear Nose Throat $J$ 2005; 84: 274-275.

13. Kawashima Y, Kobayashi D, Ishikawa N, Kishimoto S. A case of myoepithelioma arising in accessory parotid gland. J Laryngol Otol 2002; 116: 474-476.

14. Isogai R, Kawada A, Ueno K, Aragane Y, Tezuka T. Myoepithelioma possibly originating from the accessory parotid gland. Dermatology 2004; 208: 74-78.

15. Gomes M, Pepe G, Bomanji J, Al-Salihi O, Du Y, Gacinovic S, Ell P. High-grade mucoepidermoid carcinoma of the accessory parotid gland with distant metastases identified by 18F-FDG PET-CT. Pediatr Blood Cancer 2006; Sep 14.

16. Sakurai K, Urade M, Kishimoto H, Takahashi Y, Hozumi S, Yanagisawa T. Primary squamous cell carcinoma of accessory parotid gland duct epithelium: report of a case. Oral Surg Oral Med Oral Pathol Oral Radiol Endod 1998; 85: 447-451.

17. Fujimura K, Yoshida M, Sugimoto T, Kuroda $Y$ and Fujiyoshi T. Two cases of non-Hodgkin's lymphoma in the accessory parotid gland. Auris Nasus Larynx 2004; 31: 195-198.

18. Johnson FE, Spiro RH. Tumors arising in accessory parotid tissue. Am J Surg 1979; 138: 576-578.

19. Rodino W, Shaha AR. Surgical management of accessory parotid tumors. J Surg Oncol 1993; 54: 153-156. 